\title{
Bibliotheken in Frankreich: Einrichtungen, Bestände und Suchstrategien
}

Dominique Bouchery et Mareike König

\section{(2) OpenEdition}

\section{Journals}

Édition électronique

URL : http://journals.openedition.org/ifha/7889

DOI : $10.4000 /$ ifha.7889

ISSN : 2198-8943

Éditeur

IFRA - Institut franco-allemand (sciences historiques et sociales)

Référence électronique

Dominique Bouchery und Mareike König, «Bibliotheken in Frankreich: Einrichtungen, Bestände und Suchstrategien », Revue de l'IFHA [Online], HS | 2014, Online erschienen am: 01 September 2014, aufgerufen am 01 Mai 2019. URL : http://journals.openedition.org/ifha/7889 ; DOI : 10.4000/ifha.7889

Ce document a été généré automatiquement le 1 mai 2019.

CC BY-SA 3.0 


\title{
Bibliotheken in Frankreich: Einrichtungen, Bestände und Suchstrategien
}

\author{
Dominique Bouchery et Mareike König
}

Das französische Bibliothekswesen ist historisch bedingt von starkem Zentralismus geprägt ${ }^{1}$. Darin unterscheidet es sich grundlegend vom deutschen System, dessen Unübersichtlichkeit durch den Föderalismus des Landes schon fast sprichwörtlich ist. Zwei Hauptmerkmale lassen sich als Folge des Zentralismus ausmachen: zum einen ein deutliches Übergewicht an Bibliotheken und Dokumentationszentren in der Hauptstadt Paris, zum anderen eine grundlegende Zusammenarbeit zwischen den Bibliotheken und damit einhergehend zentralisierte Angebote wie beispielsweise der universitäre Bibliotheksverbund Sudoc.

Dennoch ist auch das Bibliothekssystem in Frankreich nicht ganz leicht zu durchschauen, trifft man doch ähnlich wie in Deutschland auf eine Vielzahl unterschiedlicher Bibliothekstypen: Neben der Nationalbibliothek (BnF) und ihren Standorten seien hier Universitätsbibliotheken, regionale Bibliotheken, Stadtbibliotheken und Fachbibliotheken genannt, ob in öffentlicher oder privater Hand. Hinzu kommt, dass die Betreuung des Bibliothekswesens auf zwei Ministerien verteilt ist: Die BnF, die Bibliothèque publique d'information (BPI) sowie die öffentlichen Bibliotheken stehen unter der Schirmherrschaft des Kulturministeriums (Ministère de la Culture et de la Communication) und werden von diesem beraten, unterstützt und gesetzlich kontrolliert. Die Universitäts- und Forschungsbibliotheken fallen dagegen in das Ressort des Ministeriums für Hochschulwesen und Forschung (Ministère de l'Enseignement supérieur et de la recherche). Bibliotheken und Archive sind in Frankreich grundsätzlich getrennt. In Wirklichkeit erweist sich die Lage jedoch als wenig eindeutig und voller Ausnahmen².

Eine französische Besonderheit ist das doppelte Selbstverständnis der Bibliotheken: Auf der einen Seite beherbergen sie die Schätze des nationalen Kulturerbes, so dass sich ihre Angebote an ein begrenztes Fachpublikum wenden. Auf der anderen Seite versuchen sie, das Bildungsbedürfnis eines breiten Laienpublikums zu erfüllen. Dieses Selbstverständnis 
geht auf die Zeit der Säkularisierung und der Volksbeschlagnahmungen während der Französischen Revolution zurück. Es prägt bis heute das französische Bibliothekswesen, einschließlich der Nationalbibliothek. Diese wird nicht nur als Bibliothek, sondern auch als kulturelle Stätte betrachtet, was sich in den zahlreichen Ausstellungen und Veranstaltungen dort niederschlägt.

In diesem Artikel werden die für Historikerinnen und Historiker wichtigen französischen Bibliotheken, ihre Besonderheiten und speziellen Bestände vorgestellt. Darüber hinaus werden Suchtipps und Ratschläge für den Umgang mit französischen Katalogen und Schlagworten gegeben. Eine Hilfe für alle, die mit dem fachspezifischen Vokabular Schwierigkeiten haben, ist das deutsch-französische Online-Fachwörterbuch des Instituts für Bibliothekswissenschaft der Universität Potsdam³ .

\section{Bibliotheken und ihre Bestände im Überblick}

\section{a) Paris: Hauptstadt und Schwerpunkt der Dokumentation}

\section{Die französische Nationalbibliothek (BnF)}

Bei der französischen Nationalbibliothek (früher Bn für Bibliothèque nationale, heute BnF für Bibliothèque nationale de France) reichen anders als bei ihrem deutschen Pendant die Wurzeln bis ins Mittelalter zurück ${ }^{4}$. Den Grundstock der in jeder Hinsicht herausragenden Sammlung bildet die königliche Bibliothek von Karl V. (1368). Im Jahr 1537 wurde durch Franz I. die Pflichtabgabe (dépôt légal) von Druckwerken eingeführt. Die Sammlung der Pflichtexemplare ist und bleibt eine der wichtigsten Aufgaben der BnF. Der Großteil ihrer Buchbestände ist darauf zurückzuführen.

Standorte der BnF

Die BnF hat im Laufe der Zeit zahlreiche Umwälzungen und Veränderungen durchlebt. Den letzten großen Einschnitt stellte 1995 der Umzug in den modernen großen, aus Benutzersicht jedoch gewöhnungsbedürftigen Neubau im 13. Arrondissement von Paris dar. Das von Dominique Perrault entworfene Gebäude, genannt »Tolbiac - Site François Mitterrand«, wird optisch durch vier "Büchertürme« geprägt. Sie versinnbildlichen die vier wissenschaftlichen Abteilungen:

1. Turm der Zeit: Philosophie, Geschichte, Geisteswissenschaften,

2. Turm der Gesetze: Jura, Ökonomie, Politikwissenschaften,

3. Turm der Zahlen: Mathematik, Technik und Naturwissenschaften,

4. Turm der Buchstaben: Kunst und Belletristik.

An diesem Standort findet man den Hauptteil des Bestandes der BnF. Dort ist außerdem die Inathèque ansässig. Sie verwaltet die Pflichtabgabe des französischen Rundfunks und Fernsehens. Die Tolbiac-Bibliothek ist räumlich in zwei Bereiche unterteilt: Das Obergeschoss (Haut-de-Jardin) ist für die breite Öffentlichkeit zugänglich. Das Erdgeschoss (Rez-de-Jardin) dagegen bleibt ausschließlich den Forschenden vorbehalten.

Daneben gibt es vier weitere Standorte der BnF: Auf dem historischen Grundstück "Quadrilatère Richelieu« im 2. Arrondissement befinden sich die Fachabteilungen ( départements spécialisés) für Landkarten und Pläne, Kupferstiche, Manuskripte und Inkunabeln, Fotografien, Münzen, Medaillen und Antike, und seit 2006 auch Bühnen-, 
Film- und Zirkuswissenschaften (arts du spectacle). Diese Fachabteilungen teilen das Grundstück mit der Bibliothek des Institut national d'histoire de l'art (INHA), einer Fachbibliothek ersten Ranges zur Kunstgeschichte. Sie bedient sich des ehemaligen Lesesaals der BnF, der Salle Labrouste, obwohl sie nicht zur Nationalbibliothek gehört.

Teil der BnF ist jedoch die Arsenal-Bibliothek (im 4. Arrondissement) $)^{5}$. Ihre Bestände sind stark geschichtlich und belletristisch ausgerichtet. Sie besitzt für Historikerinnen und Historiker der Frühen Neuzeit sehr interessante Sammlungen wie z.B. das Saint-SimonArchiv, das Bastille-Archiv oder für Zeithistorikerinnen- und historiker hochwertige Bestände und Manuskripte von Schriftstellern aus dem 19. und 20. Jahrhundert. Die Museumsbibliothek der Pariser Oper ${ }^{6}$ (im 9. Arrondissement) und die MusikakademieBibliothek ${ }^{7}$ runden diese Pariser Gruppe ab. Nicht in Paris, sondern in Avignon hat das Haus Jean-Vilar seinen Sitz ${ }^{8}$. Dort findet man in erster Linie Archivalien aus dem Bereich Theatergeschichte und Inszenierung.

Bestandsaufbau und Pflichtexemplare

Die BnF sammelt Werke aus allen Bereichen der Wissenschaft. Bestände werden durch das Pflichtexemplarrecht sowie durch Tausch, Erwerbungen, Nachlässe oder Schenkungen erweitert. Das Pflichtexemplarrecht wird in Kooperation mit assoziierten Bibliotheken, darunter öffentliche Bibliotheken der verschiedenen Regionen und Fachbibliotheken, wahrgenommen'. Die BnF bekommt die Pflichtexemplare der Verlage (zwei Exemplare, von denen eines an die entsprechende Fachbibliothek weitergegeben wird) und das Pflichtexemplar der in der Region Île-de-France ansässigen Druckereien. Die öffentlichen Hauptbibliotheken bekommen das Pflichtexemplar der Druckereien in ihrer jeweiligen Region. Jedoch soll das System aus Sparmaßnahmen demnächst Schritt für Schritt eingestellt werden, so dass nur noch die BnF ein einzelnes Pflichtexemplar erhält.

Seit 2006 betrifft das Pflichtexemplarrecht auch die elektronischen Veröffentlichungen. Infolge dieses Gesetzes speichert die BnF alle Inhalte der .fr-Domain des Internets. Die Film-Pflichtabgabe wird vom Centre national de la cinématographie (CNC) in Paris betreut.

Diese erste Stufe der Zusammenarbeit wird ergänzt durch das Netz der CADISTBibliotheken (Centre d'achat et de diffusion de l'information scientifique et technique). Die CADIST bilden eine Vernetzung von Fachbibliotheken bzw. von Fachsammlungen. Sie sind nach dem deutschen Vorbild der Sondersammelgebiete aufgebaut. Dieses System existiert seit 1980 und wird vom Ministerium für Hochschulwesen gesteuert. Es gibt insgesamt 24 Sammelgebiete, die auf einer Übersichtsseite im Web aufgelistet sind ${ }^{10}$. Die Aufteilung der Sammelgebiete auf die beteiligten Bibliotheken ermöglicht eine Entlastung der BnF.

Auf ihrer Website veröffentlicht die BnF seit 2000 die nationale französische Bibliografie (Bibliographie nationale française), die im Jahr 2011 zum hundertsten Mal erstellt wurde ${ }^{11}$. Auf der Startseite kann man zunächst nach Dokumententyp auswählen (Bücher, Serien, Karten ...). Auf den Unterseiten ist die Bibliographie dann über die DeweyDezimalklassifikation inhaltlich erschlossen.

Zugang zur BnF, Kopien und Scans

Um Zugang zur Forschungsbibliothek Rez-de-Jardin der BnF zu erhalten, muss man außer einem Personalausweis auch eine Bescheinigung für das eigene Forschungsvorhaben vorweisen. Das kann ein Empfehlungsschreiben eines einladenden Instituts oder die 
Bestätigung durch den betreuenden Hochschullehrenden (auf Französisch!) sein. Außerdem muss (in der Regel) eine Adresse in Frankreich angegeben werden, deren Gültigkeit durch eine Strom- oder Telefonrechnung nachgewiesen wird. Wer nur für einen kurzen Zeitraum in Paris ist, kann das entsprechend angeben und wird dann von dieser Nachweispflicht befreit. Bei der Einschreibung wird man außerdem nach dem Forschungsthema gefragt. Sinnvoll ist es, eine Literaturliste vorzubereiten, die Titel enthält, die es ausschließlich in der BnF gibt. Ansonsten kann es passieren, dass einem mit Verweis auf andere Bibliotheken der Zutritt verweigert wird. Die Einschreibung ist nur persönlich möglich. Erfahrungsgemäß bringt das vorherige Ausfüllen der Unterlagen online über die service de pré-accréditation keine Vorteile. Einmal vor Ort muss man genau die gleiche Einschreibeprozedur durchlaufen wie ohne pré-accréditation. Die aktuellen Tarife für die Leserkarten finden sich auf der Website der $\mathrm{BnF}^{12}$.

Vor Ort können Kopien oder Scans von Büchern und Zeitschriften nach dem geltenden französischen Urheberrecht angefordert werden. In der Regel können bei Büchern, die jünger als 90 Jahre sind, bis maximal 30\% ihres Umfangs kopiert oder gescannt werden. Die Kopien oder Scans werden vom Bibliothekspersonal angefertigt und kosten derzeit $0,50 €$ pro Seite. Für die Scans ist ein USB-Stick mitzubringen. Prinzipiell gibt es die Möglichkeit, Kopien oder Scans per Internet $\mathrm{zu}$ bestellen. Unter »Reproduction des Documents « finden sich entsprechende Hinweise auf der Website der $\mathrm{BnF}^{13}$. Der gesamte Vorgang war bisher sehr langsam und dauerte teilweise weit über ein Jahr. In den letzten Monaten haben sich die Vorgänge stark beschleunigt. Vom Einholen eines Kostenvoranschlags (devis) bis zur Bereitstellung des Scans vergingen bei der letzten Bestellung keine zwei Wochen.

\section{Weitere Bibliotheken in Paris und île-de-France}

In Paris und Umgebung ist die Universitätslandschaft besonders dicht. Seit der Aufgliederung der Pariser Universität befinden sich insgesamt 17 Hochschulen in der französischen Hauptstadt und Umgebung. Folglich gibt es zahlreiche Bibliotheken und Dokumentationszentren, die an diese Universitäten angegliedert sind. Einige davon sind universal, andere thematisch disziplinär ausgerichtet. Die folgenden Einrichtungen können für Historikerinnen und Historiker von besonderem Interesse sein:

Bibliothèque Sainte-Geneviève (BSG)

Die Bibliothek Sainte-Geneviève ${ }^{14}$, am Pantheon Platz gelegen, war einst Teil der Nationalbibliothek. Sie kann sich auf einen doppelten Status berufen, da sie zugleich öffentliche Bibliothek und Universitätsbibliothek ist. Sie besitzt wertvolle Altbestände und eine bemerkenswerte Sammlung zur Skandinavistik. Im gleichen Gebäude befindet sich die Bibliothèque littéraire Jacques Doucet, die zur Sorbonne gehört und deren Bestände literarisch ausgerichtet sind.

Bibliothèque publique d'information (BPI)

Die Bibliothèque publique d'information ${ }^{15}$ ist zentral in der Hauptstadt gelegen und gliedert sich in das Centre Pompidou ein. Diese Präsenzbibliothek verfügt über neuere Bestände mit Überblickscharakter zu allen Themenbereichen. Die Freihandaufstellung der Bestände, die langen Öffnungszeiten sowie der unkomplizierte Zugang ohne Einschreibepflicht machen die BPI zu einer sehr beliebten und benutzerfreundlichen Bibliothek. Ihre Popularität hat allerdings lange Wartezeiten beim Einlass zur Folge.

Bibliothèque de documentation internationale contemporaine (BDIC) 
Die Bibliothèque de documentation internationale contemporaine ${ }^{16}$ spielt eine besondere Rolle im Bereich der Zeitgeschichte. Sie ist derzeit auf zwei Standorte verteilt: In Nanterre befindet sich eine Präsenzbibliothek, die auch Archivalien, Bestände und Nachlässe aus privater Herkunft enthält ${ }^{17}$. Im Invalidendom befindet sich das Museum der BDIC mit Sammlungen von Fotografien, Plakaten, Gemälden und Objekten. Dort werden einmal im Jahr Ausstellungen zu wechselnden Themen der Zeitgeschichte gezeigt.

Die BDIC betreut die Sondersammelgebiete "Internationale Beziehungen" und »Zeitgeschichte«. Die Bibliothek wurde während des Ersten Weltkriegs mit dem Auftrag eröffnet, diesen noch laufenden Krieg $\mathrm{zu}$ dokumentieren. Ihre umfangreichen Sammlungen nicht zuletzt an grauer Literatur machen die BDIC in Frankreich zur ersten Anlaufstelle für Forschende zu diesem Konflikt. Im Rahmen des 100. Jahrestags des Ersten Weltkriegs wurden zahlreiche Bestände der BDIC digitalisiert und online zur Verfügung gestellt $^{18}$. Hervorzuheben sind auch ihre Bestände zum Zweiten Weltkrieg, zur Zwischenkriegszeit, zur ehemaligen UdSSR sowie zum Kalten Krieg. Die BDIC ist darüber hinaus eine anerkannte Einrichtung für Sozialgeschichte (Protestbewegungen, Menschenrechte) und Mitglied des Collectif des centres de documentation en histoire ouvrière et sociale (CODHOS) sowie der International labour history association (ILHA). Die BDIC arbeitet unter anderem mit der Bibliothek der Universität Paris $8^{19}$ zusammen, die das Sondersammelgebiet »DDR - neue Bundesländer« betreut. Ende 2014 wird die neue digitale Bibliothek der BDIC online gehen.

\section{Bibliothek des Deutschen Historischen Instituts Paris}

Eine wichtige Anlaufstelle für Historikerinnen und Historiker ist auch die Bibliothek des Deutschen Historischen Instituts Paris (DHIP) ${ }^{20}$, zentral im 3. Arrondissement gelegen. Sammelschwerpunkt ist die deutsche und französische Geschichte, insbesondere Regionalgeschichte, von der Antike bis zur Gegenwart. Da die Bestände analog zu den Forschungsprojekten des Instituts aufgebaut werden, sind nicht alle Themen gleichermaßen gut vertreten. Eine hervorragende Sammlung findet sich zur Hagiographie und Sozialgeschichte des Frühmittelalters, zum Herzogtum Burgund, zur Adelsgeschichte in der Frühen Neuzeit, zur Geschichte der Französischen Revolution sowie zu Frankreich unter deutscher Besatzung im Zweiten Weltkrieg. Vor Ort steht man mit Rat und Tat in bibliothekarischen Fragen zur Seite und ist ggf. mit Empfehlungsschreiben behilflich, die den Zugang zu den französischen Bibliotheken und Archiven erleichtern.

Weitere Sondersammelgebietsbibliotheken (CADIST)

In Paris haben die folgenden Bibliotheken einen thematischen Sondersammelauftrag mit Bezug zur Geschichte: Die Bibliothek der Sorbonne ${ }^{21}$ (Bibliothèque interuniversitaire de la Sorbonne) ist für die Antike zuständig. Gleichzeitig betreut sie auch die Frühe Neuzeit ( histoire moderne, 15.-18. Jahrhundert). Die bereits erwähnte Bibliothek der INHA ${ }^{22}$ ist CADIST für Kunstgeschichte und Archäologie, die Bibliothek des naturkundlich ausgerichteten Musée de l'homme betreut das Sondersammelgebiet Vorgeschichte und Paläontologie ${ }^{23}$.

\section{Fachliche Hochschulbibliotheken}

Darüber hinaus gibt es weitere fachlich spezialisierte Hochschulbibliotheken aus dem Bereich der Geschichte: die Bibliothek des Centre national des Arts et Métiers (CNAM) ${ }^{24}$ mit Beständen zu Technikwissenschaftsgeschichte, die Bibliothek der École Centrale ${ }^{25}$ in Châtenay-Malabry mit einem Schwerpunkt in Ingenieurwissenschaften, die Bibliothek 
der École normale supérieure-Rue d'Ulm (ENS) ${ }^{26}$ ebenfalls zu Mathematik, Naturwissenschaften und Geisteswissenschaften, die Bibliothek des Collège de France ${ }^{27}$ mit Beständen zu Philologie, Ethnologie, Orientalistik und die Bibliothek von Sciences Po 28, CADIST für die Politikwissenschaft. Diese Bibliotheken gehören oftmals zu traditionsreichen Einrichtungen und verfügen über wertvollen Altbestand. Teilweise kann man dort Bestände ausleihen, z.B. in der Bibliothek von Sciences Po. Weiterführende Informationen finden sich auf den in den Anmerkungen angegebenen Websites der Einrichtungen.

Eine weitere wichtige Einrichtung für Historikerinnen und Historiker ist die sozialwissenschaftliche Bibliothek der Pariser Fondation Maison des sciences de l'homme $(\mathrm{FMSH})^{29}$. Diese wurde auf Initiative von Fernand Braudel und Clemens Heller gegründet und ist derzeit provisorisch im 13. Arrondissement in der Nähe der BnF untergebracht. Die Bibliothek ist geistes- und sozialwissenschaftlich mit internationalem Blickwinkel ausgerichtet und legt Wert auf Inter- und Pluridisziplinarität. Durch die traditionelle enge Anbindung an die École des hautes études en sciences sociales (EHESS) bietet sie gerade für Historikerinnen und Historiker wertvolle Bestände.

Bibliotheken politischer Einrichtungen, Ministerien und Archive

Eine weitere Gruppe der Pariser Einrichtungen bilden Bibliotheken, die politischen Institutionen zugeordnet sind. Dazu gehören die Bibliothek des Senats ${ }^{30}$ (Bibliothèque du Sénat) und die Bibliothek der Assemblée nationale ${ }^{31}$ (Bibliothèque de l'Assemblée nationale). $\mathrm{Zu}$ erwähnen sind auch die Bibliotheken der Archive: allen voran die Bibliothek des Außenministeriums mit 450.000 Bänden vor allem zu Diplomatiegeschichte und Außenpolitik ${ }^{32}$ sowie die Bibliothek des Militärarchivs in Vincennes mit insgesamt 500.000 Medien zur Militärgeschichte ${ }^{33}$. Die Bibliotheken sind nach Anmeldung frei zugänglich.

Bibliotheken des Kulturerbes

Die Bibliothek der französischen Akademie $^{34}$ und die Bibliothek Mazarine ${ }^{35}$ stammen aus dem 17. Jahrhundert und zählen zu den ältesten öffentlichen Bibliotheken Frankreichs. Beide verfügen über bemerkenswerte Altbestände in Literatur- und Buchgeschichte sowie über Manuskripte und Autographen. Sie sind nach Anmeldung frei zugänglich.

Fachbibliotheken zur Pariser Stadtgeschichte

Die Fachbibliotheken ${ }^{36}$ der Stadt Paris sind von Interesse, wenn man sich für die Geschichte der französischen Hauptstadt insbesondere des 19. und 20. Jahrhunderts und Themen wie Architektur, Kulturgeschichte oder Verwaltung interessiert. Dazu gehören die Bibliothèque historique de la ville de Paris (BHVP), im Marais gelegen, die Bibliothèque de l'hôtel de ville de Paris (BHdV) mit Beständen zur Verwaltungsgeschichte der Stadt - beide ohne eigene Website, der Katalog ist über die zentrale Seite der Fachbibliotheken erreichbar - und die Bibliothek Forney ${ }^{37}$ für Grafik und Kunst.

Fachbibliotheken von Museen

In den letzten Jahren sind einige Fachbibliotheken in neuen Museen eröffnet worden. Die schon etwas ältere Bibliothek des Institut du monde arabe ${ }^{38}$, die Mediathek des neuen Museums Branly ${ }^{39}$ (CADIST für Ethnologie) und die Mediathek der Cité nationale de l'histoire de l'immigration ${ }^{40}$ zur Geschichte der Einwanderung gehören zu diesen Neugründungen ${ }^{41}$.

Weitere Bibliotheken 
Mit dieser Aufzählung erschöpfen sich für historisch interessierte Forschende die Bibliotheken in Paris natürlich noch nicht. So können die Bibliothèque Franciscaine des Capucins $^{42}$ oder die Bibliothèques de l'Institut Catholique de Paris ${ }^{43}$ für diejenigen interessant sein, die zur Religions- oder Kirchengeschichte arbeiten. Weitere Bibliotheken finden sich im zentralen Verzeichnis der Dokumentations- und Informationseinrichtungen Frankreichs »Répertoire national des bibliothèques et centres de documentation« (RNBCD) im Catalogue collectif de France ${ }^{44}$.

\section{b) Bibliotheken in anderen Regionen Frankreichs}

\section{Besondere Stadtbibliotheken: BMC und BMVR}

Manche Stadtbibliotheken in Frankreich besitzen historische und wertvolle Bestände. Sie werden als Bibliothèques municipales classées (BMC) bezeichnet. Derzeit gehören landesweit 55 Einrichtungen dazu ${ }^{45}$, u. a. die Stadtbibliotheken in Aix-en-Provence, Lyon, Bordeaux, Lille, Versailles, Moulins (Allier) und Saint-Omer (Pas-de-Calais). Die Bibliothek in Lyon, im 16. Jahrhundert gegründet und mit einem Bestand von über 3,8 Millionen Bänden, ist darunter die bedeutendste Einrichtung ${ }^{46}$. Sie war die erste öffentliche Bibliothek in Frankreich, die ein groß angelegtes Digitalisierungsprojekt gestartet hat. Ihre digitale Bibliothek numelyo ${ }^{47}$ enthält u. a. 55 Handschriften aus der Zeit der Karolinger und Merowinger.

Die Bezeichnung Bibliothèques municipales à vocation régionale (BMVR) umfasst zwölf große und moderne Stadtbibliotheken, deren Ausstrahlung über ihren eigentlichen Standort hinausgeht. Sie wurden in den 1990er Jahren gegründet. Dazu gehören beispielsweise die Stadtbibliotheken von Marseille, Troyes, La Rochelle und Poitiers. Manche Bibliotheken lassen sich gleichzeitig beiden Gruppen zuordnen (BMC und BMVR), so z. B. die Stadtbibliotheken in Troyes ${ }^{48}$, Reims und Nizza. Die Bibliothek in Troyes besitzt einen bemerkenswerten Altbestand, der zum großen Teil digitalisiert ist. Sie ist außerdem dank ihres Bestands aus der Bibliothek der ehemaligen Zisterzienser-Abtei Clairvaux von der UNESCO als Weltkulturerbe ausgezeichnet.

\section{Universitätsbibliotheken und SCD}

Die Universitätsbibliotheken (BU für Bibliothèques universitaires) sind republikweit über die großen und mittleren Städte verteilt. Seit den 1980er Jahren wird versucht, Bibliotheken unterschiedlicher Größe und Bedeutung zusammenzuschließen, was jedoch nicht immer von Erfolg gekrönt wird. Doch ist daraus der sogenannte SCD (Service commun de documentation) entstanden. Ziel des SCD ist es, die verschiedenen Bibliotheken wie Universitätsbibliotheken und die Außenstellen der Bibliothèques d'Unité de formation et de recherche (UFR) ${ }^{49}$ einer Universität unter einer gemeinsamen Leitung zu vereinigen. Diese Maßnahme hat weniger die Leserschaft im Blick als eine rationelle Verwaltung. Die folgenden Bibliotheken sind CADIST in den angezeigten Bereichen: SCD und Bibliothèque du Centre d'études supérieures de civilisation médiévale von Poitiers für Geschichte des Mittelalters, SCD de l'université de Provence Aix-Marseille I für den Mittelmeerraum und für Kolonialgeschichte Frankreichs. Für Sprache, Literatur und Kulturgeschichte: Straßburg für den deutschsprachigen Raum, Paris III und Lille für den englischsprachigen 
Raum, Toulouse für den spanischsprachigen Raum, Bordeaux für Lateinamerika und ehemalige portugiesische Kolonien Afrikas, Grenoble für den italienischsprachigen Raum.

Eine besondere Bibliothek beherbergt Straßburg: die Bibliothèque nationale universitaire de Strasbourg (BNUS) ${ }^{50}$. Sie genießt eine Sonderstellung, wird ihr doch aus historischen Gründen der Ehrentitel »national« verliehen. Für das Fach Germanistik spielt sie eine herausragende Rolle. In der BNUS wird die größte Sammlung an Alsatica in Frankreich aufbewahrt, für die die Bibliothek auch eine Bibliographie erstellt. Auch die BNUS arbeitet an einem großangelegten Digitalisierungsprojekt und am Aufbau ihrer digitalen Bibliothek »Numistral«, die im September 2013 online gegangen ist ${ }^{51}$.

\section{Recherchestrategien für französische Bibliotheken}

Wenn Sie vor Ort in einer Bibliothek arbeiten, ist es empfehlenswert, eine Schulung in Sachen Suchstrategie und Suchhilfe zu absolvieren. Solche Schulungen (Katalogschulung oder OPAC-Schulung) für Anfänger (initiation à la recherche documentaire) werden wie in Deutschland am Anfang eines jeden Semesters durch das Bibliothekspersonal angeboten. Im Folgenden werden zunächst Kataloge und Portale vorgestellt und anschließend einige Hinweise zur Suche in ihnen gegeben.

\section{a) Kataloge und Portale}

Obgleich die historischen Bestände in Frankreich auf eine Vielzahl von Bibliotheken verstreut sind, wird der Zugang dadurch erleichtert, dass Frankreich anders als Deutschland einen Gesamtkatalog vorweisen kann. Das Portal dieses Catalogue collectif de France $(\mathrm{CCFr})^{52}$ wird von der BnF betreut und bietet den umfassendsten Katalog für Frankreich an. Er ermöglicht eine gleichzeitige Abfrage der folgenden Kataloge: die Kataloge der BnF, die Datenbank zum Kulturerbe (Base Patrimoine) ${ }^{53}$, die Kataloge der großen Stadtbibliotheken und den Gesamtkatalog der universitären Einrichtungen Sudoc (Système universitaire de documentation). Das Portal ist nicht nur wegen seines Katalogs eine wahre Fundgrube, sondern enthält mit dem bereits erwähnten Adressverzeichnis "Répertoire national des bibliothèques et centres de documentation" (RNBCD) auch Informationen über mehr als 4.000 Dokumentations- und Bibliothekseinrichtungen aus dem ganzen Land.

\section{SUDOC}

Der Katalog Sudoc ${ }^{54}$ wird von der Agence bibliographique de l'enseignement supérieur $(\mathrm{ABES})^{55}$ betreut. Dabei handelt es sich um einen gemeinsamen Verbundkatalog der allgemeinen sowie der Fach- und Universitätsbibliotheken ${ }^{56}$. Im Sudoc werden außerdem die Pflichtexemplare der Dissertationen angezeigt, die anders als in Deutschland nicht unbedingt veröffentlicht werden müssen. Die bibliographischen Beschreibungen werden durch die jeweilige Universität eingetragen. Manche Doktorarbeiten werden als elektronische Fassungen im Volltext angeboten. 2011 wurde die Datenbank Theses.fr ${ }^{57}$ aufgebaut, in der alle ab 1985 abgeschlossenen sowie die aktuell laufenden Doktorarbeiten aller Disziplinen ganz Frankreichs verzeichnet werden. Der Provider TEL ${ }^{58}$ , finanziert vom CNRS, fördert die Selbstarchivierung von Dissertationen im Open Access und bietet auf seiner Website den Zugang zu Dissertationen im Volltext an. 


\section{Regionale Portale}

In Frankreich gibt es regionale Portale, die häufig mit digitalen Bibliotheken verbunden sind. Diese sind insbesondere bei Forschungen zu einem bestimmten geographischen Gebiet nützlich. Beispiele für diese regionalen Portale sind Lectura ${ }^{59}$ für die Region RhôneAlpes, Normannia ${ }^{60}$ für die Normandie, Alsatica ${ }^{61}$ für das Elsass, Revodoc ${ }^{62}$ für das Departement Val d'Oise oder Cedric im Departement Cantal ${ }^{63}$.

Da noch nicht alle Kataloge der Bibliotheken vollständig auf EDV umgestellt wurden, ist es in einigen Fällen notwendig, neben den Online-Katalogen vor Ort auch die Zettelkataloge und teilweise sogar alte Zugangsbücher durchzusehen. Im Folgenden einige Hinweise und Tipps für die Online-Suche.

\section{b) Suche und Lokalisierung von Zeitschriften}

In Frankreich gibt es kein Pendant zum deutschen Gesamtkatalog der Zeitschriften (Zeitschriftendatenbank, ZDB) ${ }^{64}$. Zeitschriften lokalisiert man am besten mit dem Katalog der BnF, mit dem Gesamtkatalog, der digitalen Bibliothek Gallica oder Renet ${ }^{65}$ für Online-

Ressourcen. Der Sudoc verzeichnet die Zeitschriftensammmlungen (mit Bestandsangaben) der Universitätsbibliotheken. Man wählt dafür im Online-Katalog in der einfachen Suche den Suchschlüssel »périodiques« aus. Der Suchschlüssel »Titre abrégé (périodiques)« meint die Abkürzung eines Zeitschriftentitels und ist in der Anwendung eher verwirrend und nicht zu empfehlen. Zum einen muss man die genaue Abkürzung des Titels kennen, zum anderen haben nicht alle Zeitschriften auch einen abgekürzten Titel. Die »Revue historique« hat beispielsweise die Abkürzung Rev. hist. (Paris). Sucht man nur mit »Rev. hist.« erhält man 157 Treffer. Ergänzt man den geographischen Zusatz »Paris« findet man keinen Treffer. Zu empfehlen ist die Erweiterte Suche (recherche avancée), bei der man als Dokumenttyp (type de publication) Zeitschriften (périodiques) auswählen kann.

Die Suche nach elektronischen Zeitschriften ist im Beitrag »Internet und Geschichte in Frankreich« erklärt ${ }^{66}$.

\section{c) Fernleihe und Dokumentlieferdienste}

Falls die Einrichtung, in der man gerade arbeitet, das gewünschte Dokument nicht besitzt, kann wie in Deutschland über die Bibliothek eine Fernleihe beantragt werden. In Frankreich wird dieser Dienst mit den Begriffen Prêt entre bibliothèques (PEB) für die akademische Welt (336 Einrichtungen) und Prêt inter-bibliothèques (PIB) für die öffentlichen Bibliotheken (200 Einrichtungen) bezeichnet. Beide Netze sind miteinander verknüpft.

Die Nutzungsbedingungen der Fernleihe sind von Bibliothek zu Bibliothek verschieden. In der BnF wird die Fernleihe für die ersten 20 bestellten Dokumente kostenlos durchgeführt, vorausgesetzt man ist mit dem Forscherstatus in der Bibliothek für das laufende Jahr eingetragen. In anderen Einrichtungen kann der Preis je nach Lage und Ort zwischen $6 €$ (Inland) und $8 €$ (Außenverkehr) pro Medieneinheit variieren. Bestellungen von Kopien sind nach den in Frankreich geltenden Urheberrechten ebenfalls möglich, mit Abholung vor Ort oder per Postversand. 
Einen ähnlichen Service wie Subito mit Online-Lieferung gibt es in Frankreich nicht. Eine Alternative ist der Dokumentenlieferdienst Refdoc ${ }^{67}$ von INIST-CNRS. Er bietet Zugang zu mehr als 53 Millionen Dokumenten der Fächer Naturwissenschaften, Technologie, Medizin und Geisteswissenschaften, erschienen seit dem Jahr 1823 bis heute. Die Geisteswissenschaften sind dabei eindeutig am schwächsten vertreten. Je nach Dringlichkeitsgrad können die Scans per Mail innerhalb von zwei Stunden, 24 Stunden oder sieben Tagen geliefert werden. Der Dienst ist jedoch in den letzten Monaten in Verruf geraten, weil dort Artikel teuer verkauft und zur Lieferung angeboten wurden, die es andernorts im Open Access gibt ${ }^{68}$.

\section{Suchstrategien, Dokumentenbeschreibung}

\section{a) Indexierung und Schlagwortsuche}

Die französischen Bezeichnungen für Sachschlagworte lauten vedettes matières, mots-clé und mots-sujet. Diese werden ergänzt durch die Personennormdaten und die Körperschaftsnormdaten (autorités personnes physiques et collectivités). Der zugrundliegende Thesaurus heißt im französischen Répertoire d'autorités matières encyclopédique et unifié (Rameau) ${ }^{69}$. Er enthält wie die deutsche Gemeinsame Normdatei das kontrollierte Vokabular zur inhaltlichen Erschließung der Medien und wird von fast allen französischen Bibliotheken verwendet.

\section{b) Analytische Indexierung}

Das Verzeichnis Rameau ermöglicht eine Suche nach Schlagworten (recherche par sujet) für gedruckte, audiovisuelle und ikonografische Medien, die seit 1980 von der BnF aufgenommen worden sind. Rameau wird außerdem für die Indexierung der von der Online-Bibliothek Gallica digitalisierten Medien verwendet. Auch im Verbundkatalog der universitären Einrichtungen Sudoc wird mit der Normdatei Rameau verschlagwortet.

Die inhaltliche Indexierung sollte so präzise wie möglich sein. Sie ist aber im Vergleich zu den deutschen Schlagworten meistens sehr viel weiter gefasst. Die Suche mit den Schlagworten ist - vor allem in der Kombination - nicht gerade einfach. Das französische System kennt vier Schlagworttypen, anders als im deutschen, wo es fünf gibt: Ganz vorn steht das Hauptschlagwort (tête de vedette), das ein inhaltliches Schlagwort oder ein Personenname sein kann. Dieses Hauptschlagwort kann durch drei weitere Unterschlagworte (subdivision) näher definiert sein: ein geografisches Schlagwort ( subdivision géographique), ein Zeitschlagwort (subdivision chronologique) und ein Formschlagwort (subdivision de forme).

Beispiel: Die Schlagwortkette für ein Lexikon zu den internationalen Beziehungen im 20. Jahrhundert sieht folgendermaßen aus:

Relations internationales $-20^{e}$ siècle - Dictionnaires

Ein aus einer Tagung hervorgegangener Sammelband über die Politik der EUMitgliedsländer seit dem Jahr 2000 wird wie folgt verschlagwortet:

Pays de l'Union européenne - Politique et gouvernement $-21^{\mathrm{e}}$ siècle - Congrès 
Es fallen mehrere Unterschiede zum deutschen System auf: Anders als bei den Regeln für den deutschen Schlagwortkatalog (RSWK) gibt es bei Rameau zusammengesetzte Schlagworte (Politique et gouvernement). Im Französischen werden die Schlagworte außerdem in der Regel im Plural angesetzt. Wird von dieser Regel abgewichen, dann weil sich Sinn und Gebrauch des Schlagworts verändern.

Zum Beispiel unterscheidet man »Biographie« im Singular, das ein Schlagwort für das literarische Genre ist, von »Biographies« im Plural, das als Hauptschlagwort oder Formschlagwort auf eine Person für deren Memoiren oder Lebensbeschreibung angewendet wird. Ebenso bezeichnet "Désertion« im Singular die militärische Fahnenflucht und das Schlagwort »Guerre - Désertions« die Fahnenflucht in Kriegszeiten, ohne dass genauer bestimmt wäre, welcher Krieg gemeint ist. „Désertions« im Plural wird nur in den Unterschlagworten bei bestimmten Kriegen angewendet und durch ein geographisches Unterschlagwort ergänzt, z. B. »Guerre mondiale (1939-1945) - Désertions«. Das geographische Schlagwort beschreibt dann, aus welchem Land die Deserteure stammten.

Während die Zeitschlagworte im Deutschen mit den genauen Jahreszahlen eingeleitet durch "Geschichte« angegeben werden, stehen in den französischen Katalogen zumeist die Jahrhunderte bzw. fest definierte Perioden. Das Zeitschlagwort 20. Jahrhundert wird dann verwendet, wenn das Dokument das gesamte 20. Jahrhundert oder Teile betrifft, die nicht $\mathrm{zu}$ den fest definierten chronologischen Einteilungen 1900-1945, 1945-1970, 1970-2000, 1945-1990, 1990-... gehören.

Die Anleitung für die Verschlagwortung mit Rameau ist über die Website der Nationalbibliothek zugänglich. Man kann ein Schlagwort auch im Gesamtkatalog der BnF suchen $^{70}$. Wie in Deutschland werden auch in Frankreich Belletristik und Romane nicht verschlagwortet. Die Normdaten für Personen, Körperschaften, Titel und geographische Namen werden von der BnF nach den Regeln des Rameau festgelegt und in den Sudoc eingespielt. Sie sind im Web ebenfalls über den Katalog der BnF zu recherchieren.

Über die Anwendung Idref-Autorités Sudoc ${ }^{71}$ kann man im Sudoc die Normdaten von Personen, Körperschaften, geographische Namen, Familiennamen, Autoren-Titel-Namen und Markennamen abfragen. Diese Normdaten werden wie im deutschen System für Literatur über eine Person oder über ein Werk verwendet. Über den Eintrag »Monnet Jean (1888-1979)« in Idref kommt man beispielsweise auf alle bibliographischen Einträge im Sudoc, die mit diesem Normdatensatz verbunden sind.

\section{c) Klassifikationen}

In vielen französischen Bibliotheken wird für die Signaturenvergabe die Dewey Dezimalklassifikation (DDC) verwendet. Außerdem ist teilweise die Classification décimale universelle (CDU) in Gebrauch, die ebenfalls in zehn große Gruppen unterteilt ist. Sie wird beispielsweise in der $\mathrm{BPI}^{72}$ angewandt mit einer Beschränkung auf maximal sechs Stellen. Diese Klassifikation wird jedoch immer mehr von der DDC abgelöst.

Das System der inhaltlichen Erschließung, sei es über Schlagworte oder über Klassifikationen, ist in Frankreich wie in Deutschland für Nicht-Bibliothekare oftmals nur schwer zu verstehen. Durch die Bereitstellung von Volltexten, Inhaltsverzeichnissen etc. wird jedoch die Suche in Katalogen immer einfacher, so dass man auch ohne Kenntnisse der jeweiligen Systeme und Regeln fündig werden dürfte. Hat man ein Medium gefunden, 
das der eigenen Suchanfrage entspricht, so sollte man sich die verwendeten Schlagwörter und Klassifikationen merken und damit weitersuchen.

\section{d) Sonderzeichen}

Ein Tipp noch für die Suche mit Sonderzeichen. Wenn ein Wort einen deutschen Umlaut enthält, dann darf dieser bei einer Suche in einem französischen Bibliothekskatalog nicht aufgelöst werden. Stattdessen ist der Grundbuchstabe zu verwenden. Eine Suche nach der Autorin »Schröder" auf einer französischen Tastatur also nach "Schroder«, nicht aber nach »Schroeder« auflösen. Akzente müssen in den französischen Katalogen nicht gesetzt werden. Die Suche nach dem Wort "révolution « im Titel bringt im Übrigen weniger Ergebnisse als die Suche nach »revolution«, sind doch in der zweiten Ergebnismenge auch alle englischen und deutschsprachigen Titel enthalten. Ansonsten kann man natürlich auch in den französischen Katalogen mit Trunkierungen über Platzhalter wie »?« oder »« arbeiten. Im Zweifelsfall gibt die Hilfe der Kataloge Auskunft über die verwendeten Zeichen, und natürlich kann man auch das Bibliothekspersonal vor Ort bei der Suche um Hilfe bitten.

\section{Zum Schluss}

Die Angebote der Bibliotheken bestehen natürlich nicht nur aus gedruckten Beständen und Katalogen. Das konnte hier mit der kurzen Erwähnung der regionalen Portale nur angerissen werden. Informationen und Suchhinweise für die umfangreichen Online- und Digitalisierungsprojekte in Frankreich finden sich in den Artikeln "Internet und Geschichte in Frankreich $\ll^{73}$ sowie »Archive in Frankreich ${ }^{74}$ in diesem Wegweiser.

\section{NOTES}

1. Histoire des bibliothèques françaises, 4 Bd., Paris, 1988-1992.

2. Zu diesem Punkt siehe Vincent Duclert, La séparation des bibliothèques et des archives, une spécificité française, in: Valérie Tesnière (Hg.), Histoire en bibliothèque, Editions du Cercle de la librairie, Paris 2009, S. 118-119 und den Beitrag von Florence de Peyronnet-Dryden und Matthias Nuding »Archive in Frankreich« in diesem Wegweiser (http://ifha.revues.org/7877). Vgl. auch Albert Poirot, Les archives dans les bibliothèques. Logique de service ou accident de parcours, in: Bulletin des Bibliothèques de France 2 (2001), http://bbf.enssib.fr/consulter/ bbf-2001-02-0004-001.

3. Wörterbuch Buch und Bibliothekswesen, http://www.ub.uni-potsdam.de/datenbanken/ df_glossar/startglossar.php. Alle Links wurden zuletzt am 09.04.2014 überprüft.

4. Zur BnF vgl. Daniel Renoult, Jacqueline Melet-Sanson (Hg.), La Bibliothèque nationale de France. Collections, services, publics, Editions du Cercle de la librairie, Paris 2001; Bruno Blassel, Jacqueline Melet-Sanson (Hg.), La Bibliothèque nationale de France. Mémoire de l'avenir, Paris 2006. 
5. Bibliothèque de l'Arsenal, http://www.bnf.fr/fr/la_bnf/sites/a.site_bibliotheque_arsenal.html

6. Bibliothèque de l'Opéra, http://www.bnf.fr/fr/collections_et_services/dpt_bmo/ s.collections_opera.html.

7. Département de la Musique, http://www.bnf.fr/fr/collections_et_services/dpts/ a.departement_musique.html.

8. Maison Jean-Vilar, http://www.maisonjeanvilar.org/public/accueil.html.

9. Einzelheiten über diese "Poles associés« finden sich auf der Website der BnF, http:// www.bnf.fr.

10. CADIST bei Wikipedia, http://fr.wikipedia.org/wiki/Centre_d\% 27acquisition_et_de_diffusion_de_l\%27information_scientifique_et_technique, Version 11.2.2014.

11. Bibliographie nationale française, http://bibliographienationale.bnf.fr/.

12. Website BnF, http://www.bnf.fr/fr/la_bnf/conditions_acces_tarifs/s.cond itions_acces_bib_recherche.html?first_Art=non.

13. BnF, Reproduction des Documents, http://www.bnf.fr/fr/collections_et_services/ reproductions_document.html.

14. Bibliothèque Saint-Geneviève, http://www-bsg.univ-paris1.fr/. Vgl. Yves Peyré, La bibliothèque Sainte-Geneviève à travers les siècles, Paris 2011.

15. Bibliothèque publique d'information, http://www.bpi.fr/fr/index.html.

16. BDIC, http://www.bdic.fr/.

17. Die Träger der Archive sind u.a. die Vereine Association des Déportées Internées de Ravensbrück, Ligue des Droits de l'Homme, CIMADE.

18. Vgl. Mareike König, Die digitalen Bestände zum Ersten Weltkrieg der BDIC: Feldzeitungen, Postkarten, Soldatenbriefe und mehr, in: Grande Guerre, 19.11.2013, http:// grandeguerre.hypotheses.org/1191.

19. CADIST Paris 8, http://www.bu.univ-paris8.fr/cadist-rda-nouveaux-lander.

20. Bibliothek des Deutschen Historischen Instituts Paris, http://www.dhi-paris.fr/de/home/ bibliothek/portraet.html.

21. Bibliothèque interuniversitaire de la Sorbonne, http://www.bibliotheque.sorbonne.fr/biu/; Bibliothèque de l'École française d'Athènes, http://www.efa.gr/accueil.html.

22. Bibliothèque de l'INHA, http://www.inha.fr/spip.php?rubrique21.

23. Bibliothèque du Musée de l'homme, http://www.museedelhomme.fr/ressources/ bibliotheque

24. Bibliothèque du CNAM, http://bibliotheque.cnam.fr/.

25. Bibliothèque de l'École Centrale, http://www.ecp.fr/home/Centrale_Paris/Bibliotheque.

26. Bibliothèque de l'ENS, http://www.bib.ens.fr/.

27. Bibliothèque du Collège de France, http://www.college-de-france.fr/site/bibliothequesarchives/accueil.htm.

28. Bibliothèque de la Fondation nationale des sciences politiques, http://www.sciencespo.fr/ bibliotheque/.

29. Bibliothèque de la Maison des sciences de l'homme, http://www.fmsh.fr/fr/c/238.

30. Bibliothèque du Sénat, http://www.senat.fr/; http://www.senat.fr/adresse/annuairedirection-de-la-bibliotheque-et-des-archives.html.

31. Bibliothèque de l'Assemblée nationale, http://www2.assemblee-nationale.fr/informationspratiques/archives-et-bibliotheque.

32. Bibliothèque du ministère des Affaires étrangères, http://www.diplomatie.gouv.fr/fr/leministere/archives-et-patrimoine/bibliotheques/la-courneuve-13004/

33. Bibliothèque $\mathrm{du}$ Service historique de la Défense, http:// www.servicehistorique.sga.defense.gouv.fr/Rseau-de-bibliothques.html. 
34. Bibliothèque de l'Institut de France, http://www.institut-de-france.fr/fr/patrimoine-musees/ biblioth\%C3\%A8que-de-linstitut.

35. Bibliothèque Mazarine, http://www.bibliotheque-mazarine.fr/.

36. Bibliothèques spécialisées de la ville de Paris, http://bspe-p-pub.paris.fr/Portail/Site/ ParisFrame.asp?lang=FR.

37. Bibliothèque Forney, http://equipement.paris.fr/bibliotheque-forney-18.

38. Bibliothèque de l'IMA, http://www.imarabe.org/page-sous-section/informations-pratiquesbima.

39. Médiathèque du Musée du quai Branly, http://www.quaibranly.fr/fr/enseignement/lamediatheque.html.

40. Médiathèque de la Cité nationale d'histoire de l'immigration, http://www.histoireimmigration.fr/education-et-recherche/la-mediatheque.

41. Über die Beziehungen zwischen Museum und Bibliothek siehe z. B. Odile Grandet, Bibliothèque de musée, bibliothèque dans un musée?, in: Bulletin des bibliothèques de France 4 (2007), http://bbf.enssib.fr/consulter/bbf-2007-04-0005-001.

42. Bibliothèque Franciscaine des Capucins, http://www.bibliothequefranciscaine.org/.

43. Bibliothèques de l'Institut Catholique de Paris, http://www.icp.fr/fr/Bibliotheques/Lesbibliotheques-de-l-Institut-Catholique-de-Paris.

44. Répertoire national des bibliothèques et centres de documentation, http://ccfr.bnf.fr/ portailccfr/.

45. Liste des bibliothèques municipales classées, http://www.culture.gouv.fr/public/mistral/ bibrep_fr?

ACTION=RETROUVER_TITLE\&GRP=0\&SPEC $=9 \& S Y N=1 \& I M L Y=\& M A X 1=1 \& M A X 2=1 \& M A X 3=100 \& R E Q=$ \%28\%28Biblioth\%e8que\%20municipale\%20class\%e9e\%29\%20\%3aTYPE\%20\%

29\&DOM=All\&USRNAME=nobody\&USRPWD $=4 \% 24 \% 2534 \mathrm{P}$.

46. BMC Lyon, http://www.bm-lyon.fr/.

47. Numelyo, http://numelyo.bm-lyon.fr/.

48. Médiathèque du Grand Troyes, http://www.mediatheque.grand-troyes.fr/.

49. Eine Entsprechung dieser Bibliotheken wären in Deutschland die Seminarbibliotheken.

50. Bibliothèque nationale universitaire de Strasbourg, http://www.bnu.fr/.

51. Numistral, http://www.bnu.fr/collections/numistral. Zu Numistral siehe auch Sarah Foëzon, Numistral - Die digitale Bibliothek der BNU Strassburg ist soeben online gegangen (Mittwochstipp 19), in: Franco-Fil, 9.10.2013, http://francofil.hypotheses.org/1329.

52. CCFr, http://ccfr.bnf.fr/portailccfr/. Zum CCFr siehe auch Sarah Foëzon, Der "Catalogue collectif de France«, in: Franco-Fil, 20.9.2013, http://francofil.hypotheses.org/816.

53. Base Patrimoine, http://www.culture.fr/Ressources/\%28moteur\%29/101.

54. SUDOC, http://www.sudoc.abes.fr/.

55. ABES, http://www.abes.fr/. Diese Website enthält viele allgemeine und nützliche Informationen über die Unibibliothek und ihre Dienstleistungen (Dissertationen, Fernleihe, usw.).

56. Zum Sudoc siehe auch die einleitenden Artikel von Kaja Antonowicz, Sudoc - Der Katalog der französischen Hochschulbibliotheken, Teil 1, in: Franco-Fil, 15.5.2013, http:// francofil.hypotheses.org/30 und Sven Ködel, Sudoc - Der Katalog der französischen Hochschulbibliotheken, Teil 2, in: Franco-Fil, 18.6.2013, http://francofil.hypotheses.org/360.

57. Theses, http://www.theses.fr/.

58. TEL, http://tel.archives-ouvertes.fr/.

59. Lectura, http://www.lectura.fr/.

60. Normannia, http://www.normannia.info/.

61. Alsatica, http://www.alsatica.eu/.

62. Revodoc, http://www.valdoise.fr/7553-revodoc-et-vos-bibliotheques.htm/. 
63. Cedric, http://culture.cantal.fr/.

64. Zeitschriftendatenbank ZDB, http://dispatch.opac.d-nb.de/DB=1.1/.

65. Renet, http://renet.bnf.fr/jsp/index.jsp.

66. Vgl. dazu den Beitrag von Mareike König und Annette Schläfer »Internet und Geschichte in Frankreich« in diesem Wegweiser (http://ifha.revues.org/7907).

67. Refdoc, http://www.refdoc.fr/.

68. Über den Hashtag \#inistgate konnte man die Vorwürfe in den sozialen Medien verfolgen. Eine Stellungnahme des CNRS findet sich hier: http://intranet.cnrs.fr/intranet/actus/ insist-20121019.html.

69. Rameau, http://rameau.bnf.fr/.

70. Suche nach Schlagwort im Katalog der BnF, http://catalogue.bnf.fr/jsp/recherche_autori tes_rameau.jsp?nouvelleRecherche=0\&host=catalogue.

71. Idref-Autorités, http://www.idref.fr/autorites/autorites.html.

72. BPI, Klassifikation, http://www.bpi.fr/fr/professionnels/formation_professionnelle/ documentation/classification_et_cotes.html.

73. Vgl. den Beitrag von Mareike König und Annette Schläfer »Internet und Geschichte in Frankreich« in diesem Wegweiser (http://ifha.revues.org/7907).

74. Vgl. den Beitrag von Matthias Nuding und Florence de Peyronnet-Dryden »Archive in Frankreich« in diesem Wegweiser (http://ifha.revues.org/7877).

\section{RÉSUMÉS}

Dieser Beitrag gibt einen Überblick über das universitäre Bibliothekssystem in Frankreich und stellt die wichtigsten Bibliotheken und deren Bestände in Paris und Frankreich vor. In einem zweiten Teil werden zentrale Bibliothekskataloge präsentiert sowie Tipps für die Literatursuche in französischen Katalogen gegeben. Dabei wird auch die Suche mit französischen Schlagwörtern erläutert.

\section{INDEX}

Schlüsselwörter : Frankreich, Bibliothek, Katalog, OPAC, Literaturrecherche, Fernleihe, Lieferdienst, Katalogisierung

\section{AUTEURS}

\section{DOMINIQUE BOUCHERY}

Dominique Bouchery ist zuständig für den Bestandsaufbau des »Secteur allemand« bei der Bibliothèque de documentation internationale contemporaine BDIC.

Mail: dominique.bouchery[at]bdic.fr 


\section{MAREIKE KÖNIG}

Dr. Mareike König ist Leiterin der Bibliothek und der Abteilung 19. Jahrhundert am Deutschen Historischen Institut Paris sowie Leiterin der Redaktion des deutschsprachigen Blogportals für die Geisteswissenschaften de.hypotheses.org.

Mail: mkoenig[at]dhi-paris.fr 